\title{
Ribociclib plus letrozole versus letrozole alone in patients with de novo HR+, HER2 - advanced breast cancer in the randomized MONALEESA-2 trial
}

\author{
Joyce O'Shaughnessy ${ }^{1}$ (D) Katarina Petrakova ${ }^{2} \cdot$ Gabe S. Sonke $^{3} \cdot$ Pierfranco Conte $^{4} \cdot$ Carlos L. Arteaga ${ }^{5}$. \\ David A. Cameron ${ }^{6} \cdot$ Lowell L. Hart $^{7} \cdot$ Cristian Villanueva $^{8} \cdot$ Erik Jakobsen $^{9} \cdot$ Joseph T. Beck $^{10} \cdot$ Deborah Lindquist $^{11}$. \\ Farida Souami ${ }^{12}$. Shoubhik Mondal ${ }^{13} \cdot$ Caroline Germa $^{13} \cdot$ Gabriel N. Hortobagyi $^{14}$
}

Received: 15 September 2017 / Accepted: 18 September 2017 / Published online: 21 November 2017

(c) The Author(s) 2017. This article is an open access publication

\begin{abstract}
Purpose Determine the efficacy and safety of first-line ribociclib plus letrozole in patients with de novo advanced breast cancer.

Methods Postmenopausal women with HR+, HER2- advanced breast cancer and no prior systemic therapy for advanced disease were enrolled in the Phase III MONALEESA-2 trial (NCT01958021). Patients were randomized to ribociclib ( $600 \mathrm{mg} /$ day; 3 weeks-on/1 week-off) plus letrozole ( $2.5 \mathrm{mg} /$ day; continuous) or placebo plus letrozole until disease progression, unacceptable toxicity, death, or treatment discontinuation. The primary endpoint was investigator-assessed progressionfree survival; predefined subgroup analysis evaluated progression-free survival in patients with de novo advanced breast cancer. Secondary endpoints included safety and overall response rate.

Results Six hundred and sixty-eight patients were enrolled, of whom 227 patients (34\%; ribociclib plus letrozole vs placebo plus letrozole arm: $n=114$ vs. $n=113$ ) presented with de novo advanced breast cancer. Median progression-free survival was not reached in the ribociclib plus letrozole arm versus 16.4 months in the placebo plus letrozole arm in patients with de novo advanced breast cancer (hazard ratio $0.45,95 \%$ confidence interval $0.27-0.75$ ). The most common Grade $3 / 4$ adverse events were neutropenia and leukopenia; incidence rates were similar to those observed in the full MONALEESA-2 population. Ribociclib dose interruptions and reductions in patients with de novo disease occurred at similar frequencies to the overall study population.
\end{abstract}

Conclusions Ribociclib plus letrozole improved progression-free survival vs placebo plus letrozole and was well tolerated in postmenopausal women with HR+, HER2- de novo advanced breast cancer.

Keywords Breast cancer $\cdot$ CDK inhibitor $\cdot$ Ribociclib $\cdot$ Endocrine therapy $\cdot$ De novo advanced breast cancer $\cdot$ Hormone receptor-positive

\section{Introduction}

Breast cancer diagnoses account for $25 \%$ of all newly diagnosed female cancer cases per year, affecting an estimated 1.7 million people worldwide [1]. The most common breast cancer subtype is hormone receptor-positive (HR+) disease, which constitutes $75 \%$ of all breast cancers [2]. Around $3-25 \%$ of newly diagnosed patients present with de novo

Joyce O'Shaughnessy

Joyce.OShaughnessy@USOncology.com

Extended author information available on the last page of the article
HR+ advanced breast cancer [3, 4]. Patients are classed as having de novo advanced breast cancer if they present with advanced disease without having a previous diagnosis at an earlier stage of breast cancer; this excludes patients who have received prior therapy and relapsed. Current guidelines recommend the use of first-line endocrine therapy, with or without a cyclin-dependent kinase (CDK) 4/6 inhibitor, in patients with de novo or relapsed $\mathrm{HR}+$ advanced breast cancer [5-8]. Due to a lack of exposure to systemic treatment, inherent differences may exist in the tumor biology of therapy-naïve de novo breast cancer compared with relapsed breast cancer [9], potentially contributing to the better prognosis of the de novo patient population [10]. The 
differences in clinical outcomes observed between patients with de novo and relapsed advanced breast cancer highlight the need to assess novel therapy options in this patient population [10-12].

The cyclin D-CDK4/6-inhibitor of CDK4 (INK4)-retinoblastoma $(\mathrm{Rb})$ pathway is frequently disrupted in $\mathrm{HR}+$ breast cancer [13], and has been associated with a poor clinical outcome and resistance to endocrine therapy [14]. Targeting the cyclin D-CDK4/6-INK4-Rb pathway may, therefore, present an effective strategy to enhance the efficacy of endocrine therapies. Ribociclib is an orally bioavailable, selective inhibitor of CDK4/6 [15]. Results from the Phase III MONALEESA-2 (NCT01958021) planned interim analysis demonstrated that the addition of ribociclib to letrozole significantly improved progression-free survival compared with placebo plus letrozole in patients with $\mathrm{HR}+$, human epidermal growth factor receptor 2-negative (HER2-) advanced breast cancer, with a hazard ratio of $0.56(p<0.001)$ [16]. Treatment with ribociclib plus letrozole was associated with a rapid response; $76 \%$ of patients with evaluable measurable disease had a reduction in tumor size following 8 weeks of treatment [17]. Here we report efficacy and safety results from a prospective subgroup analysis of MONALEESA-2 in patients with de novo advanced breast cancer.

\section{Methods}

\section{Study design and participants}

MONALEESA-2 is a Phase III, randomized, double-blind, placebo-controlled trial that enrolled postmenopausal women with HR+, HER2- advanced breast cancer. Full details of the study design have been published previously [16]. Briefly, patients were required to have measurable disease with at least one measurable lesion as per Response Evaluation Criteria In Solid Tumors (RECIST) v1.1 [18] or at least one predominantly lytic bone lesion. All patients had an Eastern Cooperative Oncology Group (ECOG) performance status of 0 or 1 . Patients were excluded if they had inflammatory breast cancer, central nervous system metastases, cardiac disease or Fridericia's corrected QT interval $(\mathrm{QTcF})>450 \mathrm{~ms}$, or impairment of gastrointestinal function that would have altered study drug absorption. Patients must not have received prior systemic therapy for advanced disease, except for $\leq 14$ days of letrozole or anastrozole. The use of concomitant medications with known risk of prolonging the QT interval or inducing Torsades de Pointes was prohibited.

This study was conducted in accordance with Good Clinical Practice guidelines and the Declaration of Helsinki. An independent ethics committee and institutional review boards approved the study protocol and any subsequent amendments at each participating center. A study steering committee monitored study conduct in line with the protocol. Written informed consent was obtained from all the patients.

\section{Randomization}

MONALEESA-2 patients from 223 centers in 29 countries were randomized 1:1 to receive oral ribociclib in combination with letrozole or placebo plus letrozole. Randomization was stratified according to the presence of liver and/or lung metastases. Screening and treatment allocation was performed using an interactive voice and web response system. Patients and investigators were blinded to the assigned treatment; both ribociclib and placebo were identical in label, packaging, appearance, and administration schedule. Treatment crossover from placebo to ribociclib was not permitted.

\section{Treatment and procedures}

Patients received oral ribociclib (600 mg/day, 3 weekson/1 week-off schedule, in 28-day treatment cycles) in combination with letrozole $(2.5 \mathrm{mg} /$ day, continuously) or placebo plus letrozole until disease progression, unacceptable toxicity, death, or discontinuation for any other reason. Ribociclib dose adjustments including dose interruption, reduction, and permanent discontinuation were permitted for the management of adverse events. Dose modifications of letrozole were not permitted.

Tumor response was assessed locally according to RECIST v1.1 [18]. Computed tomography/magnetic resonance imaging assessments were conducted at screening, then every 8 weeks for the first 18 months, and every 12 weeks thereafter. A whole-body bone scan was performed at baseline. Cardiac function was monitored by triplicate electrocardiograms (ECGs) performed at screening, day 15 of cycle 1 , and day 1 of cycles 2-3 in all patients; following a protocol amendment, additional ECG assessments were performed on day 1 of cycles 4-9 in all patients, and on day 1 of all subsequent cycles in patients who experienced a mean QTcF $\geq 481 \mathrm{~ms}$ prior to cycle 10 . Centralized laboratory assessments including hematology and biochemistry panels were carried out at screening, cycle 1 day 15 , and day 1 of all subsequent cycles; additional laboratory data were collected periodically throughout treatment. Adverse events were characterized throughout the study and graded according to the Common Terminology Criteria for Adverse Events (CTCAE) v4.03 [19]. 


\section{Outcomes}

The primary objective was to compare progression-free survival between the two treatment arms per local investigator assessment. Overall survival was the key secondary endpoint. Additional secondary endpoints included overall response rate, clinical benefit rate, safety, and tolerability.

\section{Statistical analysis}

This was a prespecified exploratory subgroup analysis. To compare the primary endpoint of progression-free survival between the treatment arms in the full study population, a log-rank test stratified according to the presence or absence of liver or lung metastases was utilized [16]. A stratified Cox regression analysis was used to estimate the hazard ratio and 95\% confidence intervals of progression-free survival [16]. The subgroup analysis, including Kaplan-Meier estimates and treatment effect hazard ratio estimates using an unstratified Cox regression model, was performed in patients with de novo disease. Efficacy analyses were performed in the intent-to-treat population and safety analyses were performed in all patients who received at least one dose of study treatment and had at least one post-baseline safety assessment.

\section{Results}

\section{Patient and disease characteristics}

From January 24, 2014 to March 24, 2015, 668 patients were randomized to the ribociclib plus letrozole $(n=334)$ and placebo plus letrozole $(n=334)$ arms. Among all patients randomized, 227 (34\%) presented with de novo advanced breast cancer at diagnosis; these patients were compared between treatment arms in this exploratory analysis. There was an even distribution of patients with de novo disease across both treatment arms; $114(34 \%)$ patients in the ribociclib plus letrozole arm and 113 (34\%) patients in the placebo plus letrozole arm (Table 1). Baseline characteristics of patients with de novo advanced breast cancer were well balanced across both treatment arms, except for ECOG performance status. A higher proportion of patients in the ribociclib plus letrozole arm (66\%) had an ECOG performance status of 0 , compared with $54 \%$ of patients in the placebo plus letrozole arm.

\section{Treatment}

At the interim analysis data cut-off (January 29, 2016), fewer patients with de novo disease in the ribociclib plus letrozole arm had discontinued treatment compared with those receiving placebo plus letrozole ( $30 \%$ vs. $43 \%$, respectively). The most common reason for treatment discontinuation was disease progression in both arms (Fig. 1). Patients in the ribociclib plus letrozole arm had a longer median duration of exposure to study treatment than patients who received placebo plus letrozole (14.1 and 12.8 months, respectively). The median relative dose intensity for ribociclib was $88 \%$ and the median relative dose intensity for letrozole was $100 \%$ for both treatment arms.

\section{Efficacy}

The combination of ribociclib plus letrozole prolonged progression-free survival compared with placebo plus letrozole in patients with de novo advanced breast cancer, with a hazard ratio of 0.45 (95\% CI $0.27-0.75$ ). Median progressionfree survival in the ribociclib plus letrozole arm was not reached versus 16.4 months in the placebo plus letrozole arm (Fig. 2). After 12 months, the estimated progression-free survival rates in the ribociclib plus letrozole versus placebo plus letrozole arms were $82 \%$ versus $66 \%$, respectively. The overall response rates in the intent-to-treat population were $47 \%$ in the ribociclib plus letrozole arm versus $34 \%$ in the placebo plus letrozole arm; clinical benefit rates were $83 \%$ versus $77 \%$ (Table 2). In patients with measurable disease at baseline, the overall response rates were $56 \%$ versus $45 \%$ and the clinical benefit rates were $82 \%$ versus $77 \%$ in the ribociclib plus letrozole versus placebo plus letrozole arms, respectively. Overall survival results were immature at the time of this interim analysis.

\section{Safety and tolerability}

Overall, the safety profile of ribociclib plus letrozole in patients with de novo advanced breast cancer was similar to that observed in the full MONALEESA-2 study population [16]. In the de novo subset, there was a similar incidence of all-grade adverse events of any causality in the ribociclib plus letrozole vs placebo plus letrozole treatment arms (97\% vs. $97 \%$ ). Neutropenia, nausea, and fatigue were the most common adverse events in the ribociclib plus letrozole arm (Table 3). The incidence of non-hematologic adverse events of nausea, fatigue, alopecia, vomiting, rash, pyrexia, elevated aspartate aminotransferase, and elevated alanine aminotransferase was increased by $>10 \%$ in the ribociclib plus letrozole arm compared with the placebo plus letrozole arm.

There was a higher incidence of Grade 3/4 adverse events in the ribociclib plus letrozole arm (77\%) compared with the placebo plus letrozole arm (31\%). The most common Grade $3 / 4$ adverse events $(\geq 15 \%)$ in the ribociclib plus letrozole arm were neutropenia (55\%) and leukopenia (21\%). Febrile neutropenia rates were low, occurring in $2(2 \%)$ versus 0 
Table 1 Baseline patient characteristics

\begin{tabular}{|c|c|c|}
\hline \multirow[t]{2}{*}{ Characteristic } & \multicolumn{2}{|c|}{$\begin{array}{l}\text { Patients with de novo advanced HR }+ \text {, HER2- breast } \\
\text { cancer } \\
n=227\end{array}$} \\
\hline & $\begin{array}{l}\text { Ribociclib + letrozole } \\
(n=114)\end{array}$ & $\begin{array}{l}\text { Placebo + letrozole } \\
(n=113)\end{array}$ \\
\hline Age, median (range), years & $62.5(37.0-82.0)$ & $63.0(29.0-88.0)$ \\
\hline \multicolumn{3}{|l|}{ Race, $n(\%)$} \\
\hline Caucasian & $90(79)$ & $91(81)$ \\
\hline Asian & $10(9)$ & $11(10)$ \\
\hline Black & $6(5)$ & $4(4)$ \\
\hline Other/unknown & $8(7)$ & $7(6)$ \\
\hline \multicolumn{3}{|c|}{ ECOG performance status, $n(\%)$} \\
\hline 0 & $75(66)$ & $61(54)$ \\
\hline 1 & $39(34)$ & $52(46)$ \\
\hline \multicolumn{3}{|c|}{ Disease stage at study entry, $n(\%)$} \\
\hline III & $1(1)$ & $1(1)$ \\
\hline IV & $113(99)$ & $112(99)$ \\
\hline \multicolumn{3}{|c|}{ Number of metastatic sites, $n(\%)$} \\
\hline 0 & $1(1)$ & 0 \\
\hline 1 & $33(29)$ & $36(32)$ \\
\hline 2 & $37(32)$ & $38(34)$ \\
\hline$\geq 3$ & $43(38)$ & $39(35)$ \\
\hline \multicolumn{3}{|l|}{ Site of metastases, $n(\%)$} \\
\hline Breast & $7(6)$ & $9(8)$ \\
\hline Bone & $88(77)$ & $85(75)$ \\
\hline Bone only & $28(25)$ & $24(21)$ \\
\hline Visceral $^{\mathrm{a}}$ & $53(46)$ & $55(49)$ \\
\hline Lymph nodes & $61(54)$ & $56(50)$ \\
\hline Other & $15(13)$ & $8(7)$ \\
\hline
\end{tabular}

ECOG Eastern Cooperative Oncology Group, HER2- human epidermal growth factor receptor 2-negative, $H R+$ hormone receptor-positive

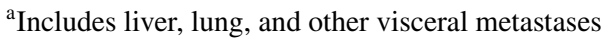

patients with de novo disease treated with ribociclib plus letrozole vs placebo plus letrozole. QTcF prolongation $>500 \mathrm{~ms}$ was not reported in any patients with de novo disease in either treatment arm. Adverse events were the most common reason for dose interruptions and reductions. At least one dose interruption due to an adverse event occurred in $75(66 \%)$ patients in the ribociclib plus letrozole arm and $17(15 \%)$ patients in the placebo plus letrozole arm. At least one dose reduction due to an adverse event occurred in 55 (48\%) patients in the ribociclib plus letrozole arm and 6 (5\%) patients in the placebo plus letrozole arm. Neutropenia was the most frequent adverse event requiring ribociclib dose interruption or dose reduction (56 [49\%] patients).

\section{Discussion}

This predefined subgroup analysis of the MONALEESA-2 trial demonstrates that postmenopausal women with de novo
HR+, HER2 - advanced breast cancer at diagnosis who received ribociclib in combination with letrozole had prolonged progression-free survival compared with those who received placebo plus letrozole, with an approximate 55\% reduction in the risk of progression (hazard ratio 0.45, 95\% CI 0.27-0.75). Median progression-free survival was not reached in the ribociclib plus letrozole arm, compared with 16.4 months in the placebo plus letrozole arm. Progressionfree survival benefit with the addition of ribociclib to letrozole was consistent with that observed in the MONALEESA-2 intent-to-treat population, where a hazard ratio of 0.56 (95\% CI $0.43-0.72, p<0.001$ ) was observed [16]. Ribociclib plus letrozole was also associated with improved overall response and clinical benefit rates versus placebo plus letrozole in patients with de novo disease.

Enhanced treatment benefits in patients with de novo advanced disease have previously been described with the CDK4/6 inhibitor palbociclib in combination with letrozole in the PALOMA-2 trial [20]. The FALCON study recently 
Fig. 1 MONALEESA-2 trial profile (CONSORT diagram)

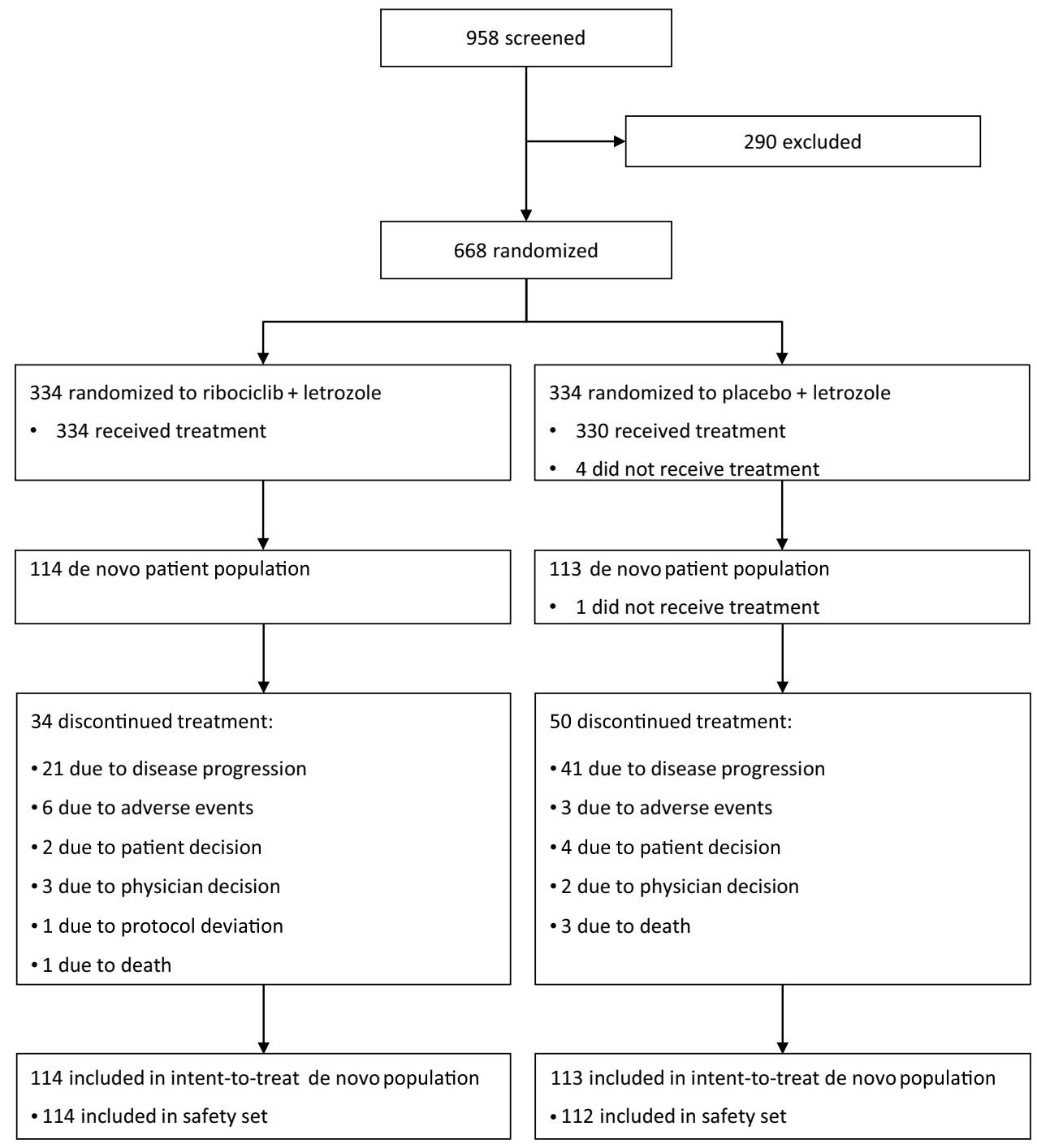

Fig. 2 Kaplan-Meier analysis of locally assessed progressionfree survival in patients with de novo advanced breast cancer in the MONALEESA- 2 trial. $C I$ confidence interval

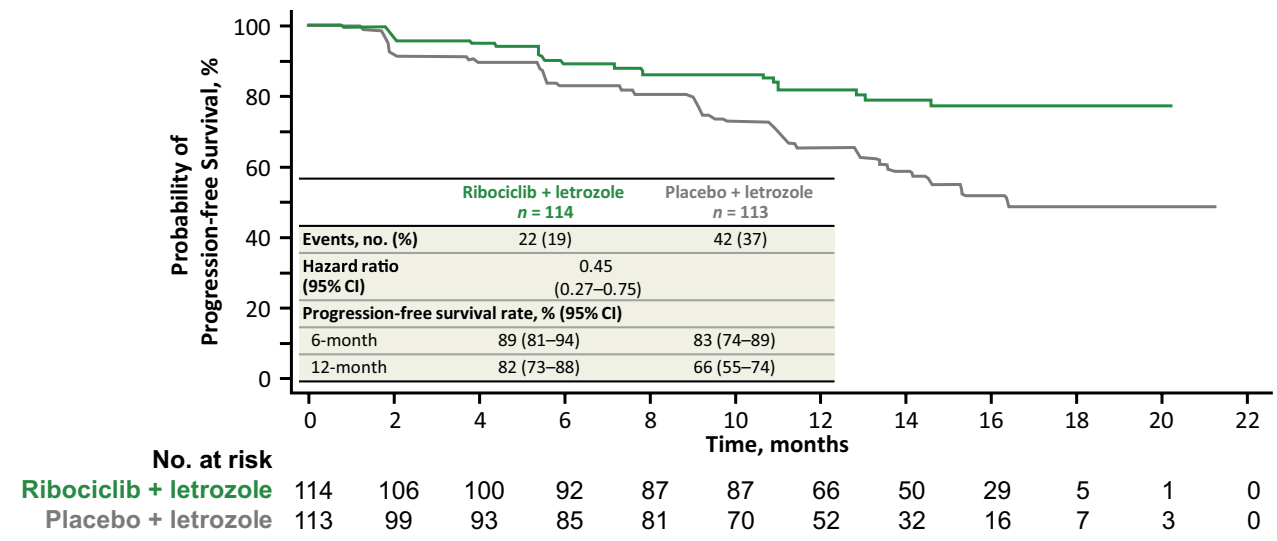

reported improved efficacy for first-line fulvestrant over anastrozole, with a hazard ratio of 0.80 (95\% CI 0.64-0.99) in a largely de novo patient population [21]. Median progression-free survival was 16.6 months in patients who received fulvestrant therapy compared with 13.8 months in the anastrozole arm; both values are in line with the median progression-free survival of 16.4 months reported herein for placebo plus letrozole-treated patients with de novo advanced disease. 
Table 2 Summary of best overall response per RECIST v1.1

\begin{tabular}{|c|c|c|}
\hline & \multicolumn{2}{|c|}{$\begin{array}{l}\text { Patients with de novo advanced HR+, } \\
\text { HER2 - breast cancer } \\
n=227\end{array}$} \\
\hline & Ribociclib + letrozole & Placebo + letrozole \\
\hline All patients, $n$ & 114 & 113 \\
\hline \multicolumn{3}{|l|}{ Confirmed BOR, $n(\%)$} \\
\hline $\mathrm{CR}$ & $2(2)$ & $1(1)$ \\
\hline PR & $52(46)$ & $37(33)$ \\
\hline SD & $36(32)$ & $34(30)$ \\
\hline NCRNPD & $16(14)$ & $26(23)$ \\
\hline $\mathrm{PD}$ & $3(3)$ & $9(8)$ \\
\hline Unknown & $5(4)$ & $6(5)$ \\
\hline $\begin{array}{l}\mathrm{ORR}^{\mathrm{a}}, n(\%) \\
{[95 \% \mathrm{CI}]}\end{array}$ & $\begin{array}{l}54(47) \\
{[38-57]}\end{array}$ & $\begin{array}{l}38(34) \\
{[25-42]}\end{array}$ \\
\hline $\begin{array}{l}\mathrm{CBR}^{\mathrm{b}}, n(\%) \\
{[95 \% \mathrm{CI}]}\end{array}$ & $\begin{array}{l}95(83) \\
{[77-90]}\end{array}$ & $\begin{array}{l}87(77) \\
{[69-85]}\end{array}$ \\
\hline $\begin{array}{l}\text { Patients with } \\
\text { measurable disease } \\
\text { at baseline, } n\end{array}$ & 96 & 83 \\
\hline \multicolumn{3}{|l|}{ Confirmed BOR, $n(\%)$} \\
\hline CR & $2(2)$ & 0 \\
\hline PR & $52(54)$ & $37(45)$ \\
\hline SD & $36(38)$ & $34(41)$ \\
\hline PD & $2(2)$ & $8(10)$ \\
\hline Unknown & $4(4)$ & $4(5)$ \\
\hline $\begin{array}{l}\mathrm{ORR}^{\mathrm{a}}, n(\%) \\
{[95 \% \mathrm{CI}]}\end{array}$ & $\begin{array}{l}54(56) \\
{[46-66]}\end{array}$ & $\begin{array}{l}37(45) \\
{[34-55]}\end{array}$ \\
\hline $\begin{array}{l}\mathrm{CBR}^{\mathrm{b}}, n(\%) \\
{[95 \% \mathrm{CI}]}\end{array}$ & $\begin{array}{l}79(82) \\
{[75-90]}\end{array}$ & $\begin{array}{l}64(77) \\
{[68-86]}\end{array}$ \\
\hline
\end{tabular}

$B O R$ best overall response, $C B R$ clinical benefit rate, $C I$ confidence interval, $C R$ complete response, HER2- human epidermal growth factor receptor 2-negative, $H R+$ hormone receptor-positive, $N C R N P D$ neither complete response nor progressive disease (for non-measurable disease at baseline), $O R R$ overall response rate, $P D$ progressive disease, $P R$ partial response, $S D$ stable disease

${ }^{\mathrm{a}} \mathrm{CR}+\mathrm{PR}$

${ }^{\mathrm{b}} \mathrm{CR}+\mathrm{PR}+(\mathrm{SD}+\mathrm{NCRNPD} \geq 24$ weeks $)$

CDK4/6 inhibitor combinations have demonstrated significantly extended progression-free survival in patients who received no prior systemic therapy for advanced disease in the MONALEESA-2 [16] and PALOMA-2 [22] trials, highlighting the utility of this treatment strategy in the first-line setting for advanced HR+, HER2- breast cancer. Recent data have also shown promising results for CDK4/6 inhibitor-based regimens in patients with $\mathrm{HR}+$, HER 2- breast cancer who received prior single-agent endocrine therapy. Abemaciclib in combination with fulvestrant extended progression-free survival compared with fulvestrant monotherapy, [23] and palbociclib plus fulvestrant also improved progression-free survival compared with fulvestrant plus placebo [24]. Data from these studies
Table 3 Adverse events (any grade; $\geq 15 \%$ in either arm), regardless of relationship to study drug

\begin{tabular}{|c|c|c|c|c|}
\hline \multirow{3}{*}{$\begin{array}{l}n(\%) \\
\text { Grade }\end{array}$} & \multicolumn{4}{|c|}{$\begin{array}{l}\text { Patients with de novo advanced HR+, HER2- } \\
\text { breast cancer } \\
n=226\end{array}$} \\
\hline & \multicolumn{2}{|c|}{$\begin{array}{l}\text { Ribociclib + letrozole } \\
(n=114)\end{array}$} & \multicolumn{2}{|c|}{$\begin{array}{l}\text { Placebo + letrozole } \\
\left(n=112^{\mathrm{a}}\right)\end{array}$} \\
\hline & All grade & Grade $3 / 4$ & All grade & Grade 3/4 \\
\hline Any adverse event & $111(97)$ & $88(77)$ & $109(97)$ & $35(31)$ \\
\hline Neutropenia $^{\mathrm{b}}$ & $80(70)$ & $63(55)$ & $5(4)$ & $1(1)$ \\
\hline Nausea & $55(48)$ & $1(1)$ & $29(26)$ & 0 \\
\hline Fatigue & $48(42)$ & $1(1)$ & $30(27)$ & $1(1)$ \\
\hline Alopecia & 45 (39) & - & $17(15)$ & - \\
\hline Leukopenia $^{c}$ & $36(32)$ & $24(21)$ & 0 & 0 \\
\hline Diarrhea & $32(28)$ & $2(2)$ & $24(21)$ & 0 \\
\hline Vomiting & $29(25)$ & $2(2)$ & $17(15)$ & 0 \\
\hline Anemia $^{\mathrm{d}}$ & $28(25)$ & $2(2)$ & $4(4)$ & $3(3)$ \\
\hline $\operatorname{Rash}^{\mathrm{e}}$ & $27(24)$ & $2(2)$ & $11(10)$ & 0 \\
\hline Arthralgia & $25(22)$ & 0 & $37(33)$ & 0 \\
\hline Back pain & $25(22)$ & $1(1)$ & $22(20)$ & 0 \\
\hline Headache & $25(22)$ & $1(1)$ & $24(21)$ & 0 \\
\hline Constipation & $24(21)$ & $1(1)$ & $18(16)$ & 0 \\
\hline Hot flush & $24(21)$ & 0 & $27(24)$ & 0 \\
\hline Decreased appetite & $22(19)$ & $2(2)$ & $21(19)$ & $1(1)$ \\
\hline Hypertension & $20(18)$ & $15(13)$ & $16(14)$ & $13(12)$ \\
\hline Pyrexia & $20(18)$ & $1(1)$ & $6(5)$ & 0 \\
\hline AST increased & $19(17)$ & $7(6)$ & $4(4)$ & 0 \\
\hline Cough & $17(15)$ & 0 & $19(17)$ & 0 \\
\hline
\end{tabular}

AST aspartate aminotransferase, HER2- human epidermal growth factor receptor 2-negative, $H R+$ hormone receptor-positive

${ }^{a}$ One patient in the placebo plus letrozole arm was randomized but did not receive study treatment

${ }^{\mathrm{b}}$ Includes neutropenia, neutrophil count decreased, and granulocytopenia

${ }^{\mathrm{c}}$ Includes leukopenia and white blood cell count decreased

${ }^{\mathrm{d}}$ Includes anemia, hemoglobin decreased, and anemia macrocytic

${ }^{\mathrm{e}}$ Includes rash and rash maculopapular

demonstrate the potential for CDK4/6 inhibitor-based combinations as second-line therapies for patients with advanced $\mathrm{HR}+$, HER2- breast cancer who have received prior single-agent endocrine therapy. Further research is required to determine the optimal treatment sequence for CDK4/6 inhibitors in advanced HR+, HER2- breast cancer, particularly since current data in the second-line setting are from patients who have received no prior CDK4/6 inhibitor-based therapy. Final overall survival analyses are needed to determine whether a potential long-term benefit is observed with the addition of a CDK4/6 inhibitor to first-line endocrine therapy, and results are eagerly awaited.

The adverse events associated with the combination of ribociclib plus letrozole in patients with de novo 
disease were consistent with those observed in the overall MONALEESA-2 study population [16] and the safety profile of other CDK4/6 inhibitors [22, 24]. The hematologic toxicities of neutropenia, leukopenia, and anemia were among the most frequent adverse events in the ribociclib plus letrozole arm, consistent with the known on-target effect of CDK4/6 inhibitors on hematologic precursors in the bone marrow [25]. Grade 3/4 neutropenia was common in patients with de novo disease-receiving ribociclib; however, the condition was rapidly reversible upon dose reduction or interruption.

In conclusion, combining ribociclib with letrozole provided clinically meaningful improvements in progressionfree survival, overall response rates, and clinical benefit rates and was well tolerated in patients with de novo advanced HR+, HER2- breast cancer. Results from this subgroup analysis of the MONALEESA-2 trial demonstrate that ribociclib plus letrozole provides a valuable first-line therapy option for women who present with de novo HR+, HER2 - advanced breast cancer at diagnosis.

Acknowledgements The authors would like to thank the patients who took part in this study and their families, as well as the staff at each study site, and all study steering committee members. Medical editorial assistance was provided by Jenny Winstanley, $\mathrm{PhD}$ (ArticulateScience Ltd.) and was funded by Novartis Pharmaceuticals Corporation. Ribociclib was discovered by Novartis Institutes for BioMedical Research in collaboration with Astex Pharmaceuticals.

Funding The study was initiated, funded, and sponsored by Novartis Pharmaceuticals Corporation. Design and conduct of the study was undertaken by the sponsor in collaboration with investigators. The study investigators and their respective research teams collected the data; Novartis Pharmaceuticals Corporation compiled the data for summation and analysis. All authors were responsible for data interpretation. The article was prepared by Dr. O'Shaughnessy in conjunction with all the authors, including employees of the sponsor. The corresponding author had final responsibility for the decision to submit the manuscript for publication.

\section{Compliance with ethical standards}

Conflicts of interest Dr. O'Shaughnessy reports advisory board member fees from Novartis. Dr. Sonke reports institutional reimbursement for steering committee activities and patient inclusion from Novartis during the conduct of the study. Dr. Cameron reports institutional reimbursement for consultancy from Novartis, Pfizer, and Lilly, outside the submitted work. Dr. Beck reports being the Principal Investigator of the CLEE011A2301 trial at Highlands Oncology Group. Dr. Souami and Dr. Germa are employees of Novartis Pharmaceuticals Corporation and hold Novartis stock options. Dr. Mondal is an employee of Novartis Pharmaceuticals Corporation. Dr. Hortobagyi reports personal fees from Novartis Pharmaceuticals Corporation during the conduct of the study; personal consultancy fees from Novartis Pharmaceuticals Corporation, Lilly USA LLC, Peregrine Pharmaceuticals Inc, F. Hoffmann-La Roche, Ltd. (Roche), Merck \& Co, and Celgene Corp outside the submitted work; and personal Scientific/Advisory Committee Member fees from Pfizer Inc., F. Hoffmann-La Roche, Ltd. (Roche), Bayer HealthCare Pharmaceuticals, and Antigen Express outside the submitted work. Dr. Petrakova, Prof. Conte, Dr. Arteaga, Dr. Hart, Dr. Villanueva, Dr. Jakobsen, and Dr. Lindquist have nothing to disclose.
Ethical approval All procedures performed in studies involving human participants were in accordance with the ethical standards of the institutional and/or national research committee and with the 1964 Helsinki Declaration and its later amendments or comparable ethical standards.

Informed consent Informed consent was obtained from all individual participants included in the study.

Open Access This article is distributed under the terms of the Creative Commons Attribution 4.0 International License (http://creativecommons.org/licenses/by/4.0/), which permits unrestricted use, distribution, and reproduction in any medium, provided you give appropriate credit to the original author(s) and the source, provide a link to the Creative Commons license, and indicate if changes were made.

\section{References}

1. International Agency for Research on Cancer (2012) GLOBOCAN 2012: Estimated cancer incidence, mortality and prevalence worldwide in 2012. http://globocan.iarc.fr/Default.aspx. Accessed 11 July 2017

2. Hosford SR, Miller TW (2014) Clinical potential of novel therapeutic targets in breast cancer: CDK4/6, src, JAK/STAT, PARP, HDAC, and PI3K/AKT/mTOR pathways. Pharmgenom Pers Med 7:203-215

3. Siegel RL, Miller KD, Jemal A (2015) Cancer statistics, 2015. CA Cancer J Clin 65:5-29

4. Miao H, Hartman M, Bhoo-Pathy N et al (2014) Predicting survival of de novo metastatic breast cancer in Asian women: systematic review and validation study. PLOS ONE 9:e93755

5. Cardoso F, Costa A, Senkus E et al (2017) 3rd ESO-ESMO international consensus guidelines for advanced breast cancer (ABC 3). Ann Oncol 28:16-33

6. Cardoso F, Costa A, Senkus E et al (2017) 3rd ESO-ESMO international consensus guidelines for advanced breast cancer (ABC 3). Breast 31:244-259

7. National Comprehensive Cancer Network (2016) NCCN clinical practice guidelines in oncology. Breast cancer, version 2.2016. https://www.nccn.org/professionals/physician_gls/pdf/breast.pdf. Accessed 11 July 2017

8. Rugo HS, Rumble RB, Macrae E et al (2016) Endocrine therapy for hormone receptor-positive metastatic breast cancer: American Society of Clinical Oncology guideline. J Clin Oncol 34:3069-3103

9. Martin M, Lopez-Tarruella S, Gilarranz YJ (2016) Endocrine therapy for hormone treatment-naive advanced breast cancer. Breast 28:161-166

10. Lobbezoo DJ, van Kampen RJ, Voogd AC et al (2015) Prognosis of metastatic breast cancer: are there differences between patients with de novo and recurrent metastatic breast cancer. Br J Cancer 112:1445-1451

11. Dawood S, Broglio K, Ensor J, Hortobagyi GN, Giordano SH (2010) Survival differences among women with de novo stage IV and relapsed breast cancer. Ann Oncol 21:2169-2174

12. Yardley DA, Kaufman PA, Brufsky A et al (2014) Treatment patterns and clinical outcomes for patients with de novo versus recurrent HER2-positive metastatic breast cancer. Breast Cancer Res Treat 145:725-734

13. Cancer Genome Atlas Network (2012) Comprehensive molecular portraits of human breast tumours. Nature 490:61-70 
14. Thangavel C, Dean JL, Ertel A et al (2011) Therapeutically activating RB: reestablishing cell cycle control in endocrine therapyresistant breast cancer. Endocr Relat Cancer 18:333-345

15. Kim S, Loo A, Chopra R et al (2013) LEE011: an orally bioavailable, selective small molecule inhibitor of CDK4/6-reactivating $\mathrm{Rb}$ in cancer. Mol Cancer Ther 12:Abstract PR02

16. Hortobagyi GN, Stemmer SM, Burris HA et al (2016) Ribociclib as first-line therapy for HR-positive, advanced breast cancer. $\mathrm{N}$ Engl J Med 375:1738-1748

17. André F, Stemmer SM, Hortobagyi GN et al (2016) Ribociclib + letrozole for first-line treatment of HR+, HER2- ABC: efficacy, safety, and pharmacokinetics. EORTC-NCI-AACR. https://doi. org/10.1016/S0959-8049(16)32619-3

18. Eisenhauer EA, Therasse P, Bogaerts J et al (2009) New response evaluation criteria in solid tumours: revised RECIST guideline (version 1.1). Eur J Cancer 45:228-247

19. National Cancer Institute Cancer Therapy Evaluation Program (2010) Common Terminology Criteria for Adverse Events (CTCAE) v4.0. https://ctep.cancer.gov/protocoldevelopment/ electronic_applications/ctc.htm. Accessed 11 July 2017

20. Finn RS, Martin M, Rugo HS et al (2016) Palbociclib and letrozole in advanced breast cancer. N Engl J Med 375:1925-1936

21. Robertson JFR, Bondarenko IM, Trishkina E et al (2016) Fulvestrant $500 \mathrm{mg}$ versus anastrozole $1 \mathrm{mg}$ for hormone receptor-positive advanced breast cancer (FALCON): an international, randomised, double-blind, Phase III trial. Lancet 388:2997-3005

22. Sledge GW Jr, Toi M, Neven P et al (2017) MONARCH 2: abemaciclib in combination with fulvestrant in women with $\mathrm{HR}+$, HER2 - advanced breast cancer who had progressed while receiving endocrine therapy. J Clin Oncol. https://doi.org/10.1200/ JCO.2017.73.7585

23. Cristofanilli M, Turner NC, Bondarenko I et al (2016) Fulvestrant plus palbociclib versus fulvestrant plus placebo for treatment of hormone-receptor-positive, HER2-negative metastatic breast cancer that progressed on previous endocrine therapy (PALOMA-3): final analysis of the multicentre, double-blind, Phase II randomised controlled trial. Lancet Oncol 17:425-439

24. Finn R, Jiang Y, Rugo $\mathrm{H}$ et al (2016) Biomarker analyses from the Phase III PALOMA-2 trial of palbociclib (P) with letrozole (L) compared with placebo (PLB) plus L in postmenopausal women with ER+/HER2 - advanced breast cancer (ABC). Ann Oncol 27(suppl 6):Abstract LBA15

25. Asghar U, Witkiewicz AK, Turner NC, Knudsen ES (2015) The history and future of targeting cyclin-dependent kinases in cancer therapy. Nat Rev Drug Discov 14:130-146

\section{Affiliations}

\section{Joyce O'Shaughnessy ${ }^{1}$ (D) Katarina Petrakova ${ }^{2} \cdot$ Gabe S. Sonke $^{3} \cdot$ Pierfranco Conte $^{4} \cdot$ Carlos L. Arteaga $^{5}$. David A. Cameron ${ }^{6} \cdot$ Lowell L. Hart $^{7}$. Cristian Villanueva ${ }^{8} \cdot$ Erik Jakobsen $^{9} \cdot$ Joseph T. Beck ${ }^{10}$. Deborah Lindquist ${ }^{11}$. Farida Souami $^{12}$. Shoubhik Mondal ${ }^{13} \cdot$ Caroline Germa $^{13} \cdot$ Gabriel N. Hortobagyi $^{14}$}

1 Texas Oncology-Baylor Charles A. Sammons Cancer Center and US Oncology Network, 3410 Worth Street, Suite 400, Dallas, TX 75246, USA

2 Masaryk Memorial Cancer Institute, Žlutý kopec 543/7, 65653 Brno, Czech Republic

3 Netherlands Cancer Institute and BOOG Study Center, IJsbaanpad 9, 1076 CV Amsterdam, The Netherlands

4 University of Padova and Istituto Oncologico Veneto, IRCCS, Via Gattamelata, 64, Padua, Italy

5 Vanderbilt-Ingram Cancer Center, 1301 Medical Center Dr \#1710, Nashville, TN 37232, USA

6 Edinburgh Cancer Centre, University of Edinburgh, Crewe Rd S, Edinburgh EH4 2XR, UK

7 Sarah Cannon Research Institute, Fort Myers, FL 33916, USA
8 University Hospital of Besançon, Jean-Minjoz University Hospital, 3 Boulevard Alexandre Fleming, 25000 Besançon, France

9 Lillebaelt Hospital, Kabbeltoft 25, 7100 Vejle, Denmark

10 Highlands Oncology Group, 3232 N Northhills Blvd, Fayetteville, AR 72703, USA

11 Arizona Oncology, US Oncology Network, 3700 W State Rte 89A, Sedona, AZ 86336, USA

12 Novartis Pharma AG, Fabrikstrasse 2, 4056 Basel, Switzerland

13 Novartis Pharmaceuticals Corporation, 1 Health Plaza, East Hanover, NJ 07936, USA

14 University of Texas MD Anderson Cancer Center, 1515 Holcombe Blvd, Houston, TX 77030, USA 